\title{
Flow Shop Scheduling Problem with Transportation Times, Two-Robots and Inputs/Outputs with Limited Capacity
}

\author{
Tarik Lamoudan, Fatima El Khoukhi, \\ Ahmed El Hilali Alaoui \\ Faculty of Sciences and Technology, Fez \\ Morocco
}

\author{
Jaouad Boukachour \\ University Institute of Technology \\ Le Havre, France
}

\begin{abstract}
In this paper we study a generalized flow-shop problem with two identical transport robots between machines. Besides transportation times for the jobs, empty moving times for the robots are taken into account. The objective is to minimize the Makespan.

An ant colony algorithm with appropriate probability transfer structures is proposed. Computational results are presented for test data arising from flow-shop benchmark instances enlarged by transportation and empty moving times. Keys words: flow shop, limited buffers, transportation times, ant colony system.
\end{abstract}

\section{Introduction}

A flow-shop problem with transportation times and two robots is a generalization of the classical flow-shop problem and may be formulated as follows: We are given $\mathrm{m}$ machines $\mathrm{M}_{1}, \ldots, \mathrm{M}_{\mathrm{m}}$ and $n$ jobs $j=1, \ldots, n$. Each job $j$ consists of $m$ operations $O_{i j}(\mathrm{i}=1, \ldots, \mathrm{m})$ which have to be processed in the order $\mathrm{O}_{1 \mathrm{j}} \rightarrow \mathrm{O}_{2 \mathrm{j}} \rightarrow \ldots \rightarrow \mathrm{O}_{\mathrm{mj}}$. Operation $\mathrm{O}_{\mathrm{ij}}$ has to be processed on machine $\mathrm{M}_{\mathrm{i}}$ without preemption for $p_{i j} \geq 0$ time units. Each machine can only process one job at a time. Additionally, transportation times are considered. They occur if a job changes from one machine to another, (i.e. if operation $O_{k j}$ is processed on machine $M_{k}$ and afterwards $O_{k+1, j}$ on machine $M_{k+1}$, a transportation time $t_{i j} \geq 0$ arises). These transportation times was may be jobdependent or job-independent $\left(t_{j k}=t_{k}\right)$. We consider the case where all transportations are done by two identical transport robots $\left\{\mathrm{R}_{1}, \mathrm{R}_{2}\right\}$ which each robot can only handle one job at a time. Thus, conflicts between transportations may arise and a job may have to wait for the robot before its transportation. We assume that empty moving times of the robot are negligible, (i.e. if the robot moves empty without carrying a job any additional times are considered). All values $p_{i j}$ and $t_{j k}$ are supposed to be non-negative Integers.

We assume that we have limited buffer spaces between the machines (input/output). Each machine $M_{k}$ has a limited output buffer where jobs processed on $M_{k}$ and waiting for the robot may be stored. The jobs are automatically transferred into this buffer and no further times for this transfer are considered. Additionally, each machine $M_{k}$ has a limited input buffer where jobs awaiting processing on $M_{k}$ may be stored. Each buffer space (input/output) can only support one task at a time.

The objective is to determine a feasible schedule which minimizes the Makespan $C_{\max }=\max _{j=1}^{n} C_{j}$, where $C_{j}$ is the finishing time of the last operation $O_{m j}$ of job j.

\section{Related work}

To describe special cases of this problem Johann Hurink, and Sigrid Knust [8] are extended the $\alpha|\beta| \gamma$-notation of Graham et al. [7]. In the $\alpha$ field we write $R 1$ to indicate that one robot is available for all transportations. In the $\alpha$-field we add information about the transportation times:

- $\mathrm{t}_{\mathrm{jk}}$ means that the transportation times are job-dependent and machine-dependent.

- $\mathrm{t}_{\mathrm{jk}}=t_{\mathrm{k}}$ denotes machine-dependent but job-independent transportation times. In practice this situation may occur for jobs of the same size where the transportation times only depend on the travel distances between the machines.

- $\mathrm{t}_{\mathrm{jk}}=\mathrm{T}$ indicates that all transportation times are equal to a constant $\mathrm{T}$. 
If we have only $m=2$ machines, the robot always transports from M1 to M2. Therefore, the index $\mathrm{k}$ in the notation $\mathrm{t}_{\mathrm{jk}}$ is dropped and the transportation times are denoted by $t_{j}$. If they may take only two values T1, T2, we write $t j \in\{T 1 ; T 2\}$ in the $\beta$ field. In practice this situation may occur if we are given two types of jobs, some "big" and some "small" jobs where it takes longer to transport the big jobs than the smaller ones.

The permutation flow shop problem (PFSP) with the objective of minimizing makespan or total flow time had been proved they are NP-Complete (Garey et al. [6]).

Although in the literature, several exact methods have been tested on this kind of problem (Bansal, [1]), but they were forced to limit the size of the problem due to the fact of the explosion calculations with the nature of NP-complete problem. Several authors have worked on the complexity of flow shop problem with transportation. Many authors focused on cyclic flow-shop scheduling problems.

For the flow-shop environment with a single robot and unlimited buffer space only one complexity result is available. Kise [9] has shown that problem $F 2, R 1|t j=T| C \max$ is NP-hard in the ordinary sense. For the flow shop environment with a single server (Immediately before processing an operation $\mathrm{O}_{\mathrm{ij}}$ the corresponding machine has to be prepared for processing $\mathrm{O}_{\mathrm{ij}}$, which takes a setup time of $\mathrm{s}_{\mathrm{ij}}$ time units. During such a setup the machine is also occupied for $s_{i j}$ time units, i.e. no other job can be processed on it.), several complexity results are available in Brucker et al. [2], have proven that $\mathrm{F} 2, \mathrm{~S} 1\left|\mathrm{p}_{\mathrm{ij}}=\mathrm{p}\right| \mathrm{Cmax}$ and $\mathrm{Fm}, \mathrm{S} 1\left|\mathrm{p}_{\mathrm{ij}}=1\right| \sum \mathrm{T}_{\mathrm{j}} \forall \mathrm{m} \geq 2$ are NP-hard for two or greater than two machines, but they aren't presented a resolution methods for this kind of problems. For flow-shop problems with transportation times, limited buffer space and a single robot, some polynomially solvable special cases have been described by Panwalkar [13]. Kise et al. [10], and Stern and Vitner [16] considered the two-machine case with an additional no-wait condition which can be formulated as a specially structured traveling salesman problem.

For solving the problem two ant-colony algorithms are proposed and analyzed by [14] in order to minimize the total flow time in permutation flow shop, KC. Ying CJ. Liao [20] introduces the ant colony optimization for the first time to resolve the permutation flow-shop with minimizing the Makespane objective. Qian et al [12] proposed a hybrid algorithm based on differential evolution
(DE-based algorithm) for multi-objective flow shop scheduling with limited buffers.

This paper is organized as follows. In Section 3, the flow-shop scheduling problem with transportation times is stated and formulated and the mathematic model for the flow-shop problem with transportation constraints is proposed. In Section 4, we present a graphical representation, architecture for the workshop studied and we give a disjunctive graph representation for the problem. In section 5, the ACO (Ant Colony Optimization) algorithm proposed is detailed. In Section 6, the computational results and comparisons are provided, and we finish by a conclusion.

\section{Problem formulation and mathematic model}

\subsection{Problem formulation}

In most manufacturing and distribution systems, finished tasks are transferred from a machine to another by robots transporter. This paper investigates the flow shop architecture. We have two transporter robots $\mathrm{R}_{1}$ and $\mathrm{R}_{2}, \mathrm{~N}$ jobs $\mathrm{J} 1, \mathrm{~J} 2, \ldots, \mathrm{Jn}$ and $\mathrm{M}$ machines $\mathrm{M}_{1}, \mathrm{M}_{2}, \ldots, \mathrm{M}_{\mathrm{m}}$. Each job Jj consists of $\mathrm{M}$ operations $\quad O_{i j}, \quad \forall i=1, \ldots m$, and $\forall j=1, \ldots, n$ which have to be processed in the order $M_{1} \rightarrow M_{2} \rightarrow, \ldots, M_{m}$, without preemption on machines. The processing times of the operations of $\mathrm{Jj}$ are given by $\mathrm{p}_{\mathrm{i}, \mathrm{j}} \geq 0$. At any given time, one machine can only process one operation and one robot can only transferred one task. The finished tasks is stored in output buffer if it is available and must be transferred by one robot transporter at a time and delivered to preview machine in $\mathrm{tr}_{\mathrm{hk}} \geq 0$ units of times. Each machine $\mathrm{M}_{\mathrm{i}}$ has a limited buffer input/output and its capacity has limited in one task at a time, in such a case, after finishing processing on a machine, a job either directly has to be processed on the next machine or it has to be stored in the output buffer between the two machines. If the buffer is full, the job has to wait on its current machine and this machine is blocked for other jobs. This blocking will be remained until at least a buffer unit becomes available.

\subsection{Mathematical Model}

In this section we give the variables and data that we use in the mathematical model. 


\subsubsection{The variables and data}

$x_{i j}^{k}= \begin{cases}1 & \text { if the job } \mathrm{j} \text { preced the job } \mathrm{i} \text { on the machine } \mathrm{k} \\ 0 & \text { otherwise }\end{cases}$

$y_{r j}= \begin{cases}1 & \text { if job } \mathrm{j} \text { is carried by the robot } \mathrm{r} \\ 0 & \text { otherwise }\end{cases}$

$c_{i k}$ The start time of job i on machine $\mathrm{k}$

$t_{i k}$ The finish time of job i on machine $\mathrm{k}$

$p_{i k}$ The processing time of job i on machine $\mathrm{k}$

$t r_{h k}$ The transportation time from machine $\mathrm{h}$ to machine $\mathrm{k}$

$h_{i k}$ The start time of transportation of job $\mathrm{i}$ to machine $\mathrm{k}$

$d_{i}$ The time of availability of job $\mathrm{i}$ in the initial depot

$C_{\text {in }}^{k}$ The input capacity of machine $\mathrm{k}$

$C_{\text {ou }}^{k}$ The output capacity of machine $\mathrm{k}$

\subsubsection{The objective function}

Minimize $C_{\max }$

In the remaining of this paragraph we present the problem's constraints.

\subsubsection{The constraints}

$\left.t_{j, k+1} \geq c_{j, k}+t r_{k, k+1} \quad \forall \quad k=0, \ldots, m-1 \quad \forall j=1, \ldots, n \quad 1\right)$

A job $\mathrm{j}$ cannot start in the next machine after its transport at least.

$x_{i j}^{k}+x_{i j}^{k}=1$

Each machine can only process one job at a time.

$t_{j, k} \geq t_{i, k}+p_{i, k}-H x_{i, j}^{k} \quad \forall j, i=1, \ldots, n$

This constraint illustrates the precedence constraints between jobs on machines.

$t_{i 1} \geq d_{i}$

The job i can only begin after the date of availability in the initial depot.

$\sum_{j=1}^{n} y_{r j} \leq 1 \quad \forall \mathrm{r}=1,2$

The robot can at most transport one task at a time (the empty movements are allowed)

$\sum_{r=1}^{2} y_{r j} \leq 1 \quad \forall \mathrm{j}=1, \ldots, \mathrm{n}$

Each job can be transporter only by one robot.

$C_{\text {max }} \geq C_{j}+\operatorname{tr}_{\mathrm{mf}} \quad \forall \mathrm{j}=1, \ldots, \mathrm{n}$
This constraint ensure that the Cmax (Makespan) is greater than the finished date of the last job on the machine $M_{m}$ plus the time of transport to the final depot.

$h_{j k} \geq c_{j, k-1} \quad \forall j=1, . ., n$

The $8^{\text {th }}$ constraint assures that each job $\mathrm{j}$ cannot be transported to the next machine after its availability on the output of its current machine.

$c_{j k}=t_{j k}+p_{j k} \quad \forall j=1, . ., n \quad \forall k=1, \ldots, m$

This constraint ensure the non preemption of the tasks on the machine

\section{Graphical representation}

This paper focuses on sequencing and scheduling for a particular type of automated material handling system in cellular manufacturing: robotic cells. A robotic cell consists of an input device; a series of processing stages, each of which performs a different operation on each part in a fixed sequence; an output device; and one or more robots that transport the parts within the cell. Each stage has one or more machines that perform the processing for that stage.

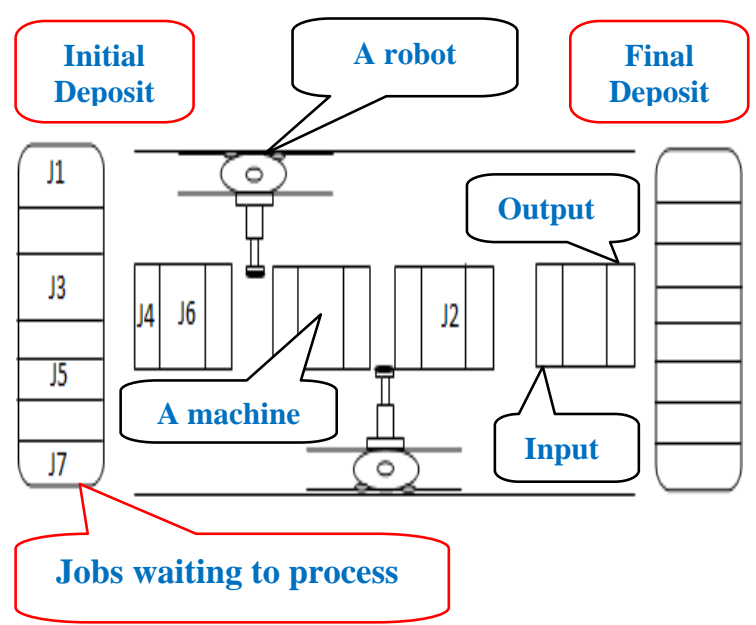

Figure 1. Flow-shop architecture

The configuration of a robotic cell (Figure 1) is to have two robots that can hold only one part by each robot, one machine per stage, and buffers spaces (input/output) can only support one task at a time for intermediate storage between the stages within the cell; each machine can hold only one part, a robotic cell is, in essence, a flowshop with two robot and on capacity for input/output buffers for each machine.

Our flow-shop system (Figure 1) is composed of a set of machines and two robots positioned on the two sides of the machines to move finished tasks from one machine to the others, by parallel movements 
throughout the mock parallel. For more details about the architecture see dawande [4].

The flow shop scheduling problem consists of the arrangement of $\mathrm{n}$ jobs on $\mathrm{m}$ machines. The processing times of $\mathrm{n}$ jobs on the machines are known in advance. This problem can be represented by a disjunctive graph. Figure 2 shows a disjunctive graph representation for the problem made of four jobs and three machines. In a disjunctive graph, circles represent jobs. Conjunctive arcs correspond to precedence constraints among the machines for the same job. Disjunctive arcs correspond to possible constraints among the jobs on the same machine. The general graph model of the flow shop with buffers can be found in the article of Nowicki [11].

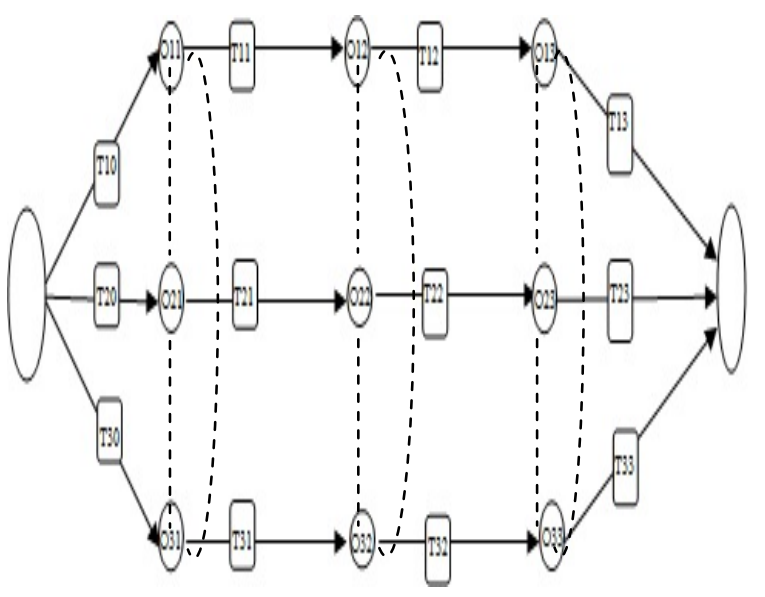

@: Input/output

$\mathbf{O}_{\mathrm{ij}}$ : Job i on machine $\mathrm{j}$

$\mathbf{T}_{\mathbf{i j}}$ : Task of transport

Figure 2. Disjonctif Graph of a Flow-Shop system with 3 jobs and 3 machines

\section{Resolution approach}

The ant colony optimization (ACO) heuristic presented by Colorni et al. [3], and later extended by several studies, used a swarm intelligence approach to solve the traveling salesman problem (TSP). R.F. Tavares Neto and M.Godinho Filho [17] are used this algorithm to solve a permutation flow shop problem with outsourcing allowed, they use new ACO approach in two sequential ACO heuristic blocks, each ACO algorithm solves a different part of the problem. The significance of an ant's move is highly dependent on the graph representation of the problem, and then to use this method to solve any problem, so first of all, we must describe this problem as a graph representation. This constructive method operates on the network structure of the graph, because the ant that is the main element of the method moves in the graph of a node to another using a transition probability which operates several heuristics information. This method was proposed by Gambardella and Dorigo [5] to solve the traveling salesman. Our ACO algorithm follows different steps:

1) Initialization step: traces of pheromone information and the parameters of heuristic are initialized.

2) Iterative step: a colony of ants determines the first jobs to be transported to the initial machine. Each ant applies repeatedly state transition rule to select the next job until a complete solution (a schedule) be built. In constructing a schedule (one solution), the information heuristic and pheromone trails are used to determine which job will be chosen.

Each robot is represented by an ant, the two ants communicate with each other during the construction of the solution. When a robot is moving to a machine to serve, then the other robot chooses other one to serve it.

\subsection{Pheromone trails}

All the jobs are positioned in the initial deposit at time $d_{i}=0$. Pheromone trail $\tau_{i j}$ indicates the sequence desire of job $\mathrm{j}$ to follow job i. Initial pheromone trails $\tau_{0}$ are relatively small quantity and same for all edges.

\subsection{Heuristic information}

The heuristic information value is also initialized at the initialization step. The use of heuristic information to direct the ants' probabilistic solution construction is important because it provides problem specific knowledge. Heuristic information used in this study is the time of process of jobs on the machines $\eta_{i j}=\frac{1}{p_{i j}}$, the time of transport of jobs between machines $t r_{h k}$ and the remaining time ( $\theta_{i j}$ ) for the total execution time of machine.

\subsection{State transition rule}

In the process of schedule forming, an ant $\mathrm{k}$ in node $\mathrm{i}$ select the node $\mathrm{j}$ to move by applying the following state transition rule: 
$j_{\text {next }}=\left\{\begin{array}{l}\arg \max \left\{\left(\tau_{i j}\right)^{\alpha} \times\left(\eta_{i j}\right)^{\beta} \times\left(\theta_{i j}\right)^{\varphi}\right\} \quad \mathrm{j} \in L C \\ 0 \quad \text { otherwise }\end{array}\right.$

Where:

- $\tau_{i j}$ is the amount of pheromone trail on edge (i,j), $\eta_{i j}=\frac{1}{p_{i j}}$ is the inverse of the execution time of job $\mathrm{i}$ on the machine $\mathrm{j}$;

- $\theta_{i j}$ is the total remaining execution time for the machine $\mathrm{j}$. It should be emphasized that the set of feasible nodes is containing in the (list of candidates);

- $\alpha$ is a parameter that allows a user to control the relative importance of pheromone trail ( $\alpha>0)$;

- $\beta$ is a parameter that determines the relative importance of the first heuristic information $\eta_{i j}(\beta>0)$;

- $\varphi$ is a parameter that determines the relative importance of the second heuristic information $\theta_{i j}(\varphi>0)$;

- $\mathrm{J}$ is a random variable selected according to the following random-proportional rule probability distribution, which is the probability of that ant $\mathrm{k}$ chooses node $\mathrm{j}$ with larger $p_{i j}$ to move from node $\mathrm{i}$ :

- $\quad$ LC is a list of candidates

The passage of an ant from a node $\mathrm{i}$ to node $\mathrm{j}$ falls

$$
p_{i j}=\left\{\begin{array}{l}
\frac{\left\{\left(\tau_{i j}\right)^{\alpha} \times\left(\eta_{i j}\right)^{\beta} \times\left(\theta_{i j}\right)^{\varphi}\right\}}{\sum_{j \in \text { list of candidates }}\left(\tau_{i j}\right)^{\alpha} \times\left(\eta_{i j}\right)^{\beta} \times\left(\theta_{i j}\right)^{\varphi}} \quad \text { if } j \in \text { LC } \\
0 \quad \text { otherwise }
\end{array}\right.
$$

into two equations: eq (1 ') and eq (2'), and from their solutions, it chooses the best one.

\subsection{Local updating rule}

While constructing a schedule, an ant decreases the pheromone trail level between selected jobs by applying the following local updating rule:

$$
\tau_{(i, j)}=\left(1-\rho_{l}\right) \tau_{(i, j)}+\rho_{l} \times \tau_{0}
$$

Where, $\tau_{0}$ is the initial pheromone level and $\rho_{l}\left(\rho_{l}>0\right)$ is the local pheromone evaporating parameter.

\subsection{Global updating rule}

Global updating rule is applied after all ants completed their schedules. Global updating provides a greater amount of pheromone trail between adjacent jobs of best schedule. The pheromone trail level is updated as follows:

$$
\tau_{i j}(k)=\rho_{g} \times \tau_{i j}(k-1)+\Delta_{\tau_{i j}}
$$

Where,

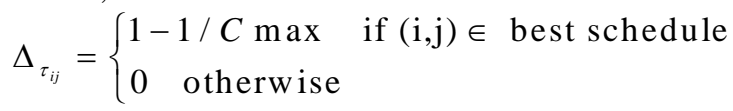

In Eq. (4'), $\rho_{g}\left(\rho_{g}>0\right)$ is the pheromone evaporating parameter of global updating and Cmax is the objective function value of the best schedule until the current iteration.

\subsection{ACO Pseudo code for classical permutation flow shop problem}

In this paragraph we present a pseudo code of the ACO algorithm.

1. Initialization: The pheromone trails, the heuristic information and the parameters are initialized.

2. Iterative loop:

2.1 A colony of ants determines starting jobs.

2.2 Each ant constructs a complete schedule:

Repeat

Apply state transition rule (2') to select the next processing job

Apply the local updating rule

Until a complete schedule is constructed

2.3 Apply the global updating rule

3. Cycle: If the maximum number of iterations is realized, then STOP;

Else go to step 2.

\section{Computational results}

The results shown in Table 1 concern the permutation flow shop problem with unlimited buffers, and no transportation time (i.e. that the sequence in which each machine processes all jobs is identical on all machines (permutation flow shop)). Our method was tested on 28 benchmark problems with 20 jobs and the number of machines varying from 5 to 20 given by Taillard [18]. There were 10 instances for each problem size. The test problem files are available via OR-Library web site (URL: http:

mscmga.ms.ic.ac.uk/jeb/orlib/\&owshopinfo.html), or 
can be downloaded from the Taillard's web site (URL: http://www.idsia.ch/ eric/)

Performance of solutions to yield using test problem is compared with MOACSA algorithm of Betul Yagmahan and Mehmet Mutlu Yenisey [19], CR(MC) algorithm of Rajendran [14] and HAMC algorithms (HAMC1, HAMC2, HAMC3) of Ravindran et al. [15].

The solution quality is measured by the mean percentage difference from Taillard's upper bound. In the Table 1 we offer a comparison between different solution methods found in the literature and our proposed resolution method (ACO) to solve the flow shop sequencing problem with transportation constraints, the computational result shown in the Table 1, represent the quality of performance of (C$\left.\mathrm{C}^{*}\right) / \mathrm{C}^{*}$ of ACO and the other methods. Where $\mathrm{C}^{*}$ is the best solution found so far and $\mathrm{C}$ is the best solution obtained by each method.

we find that our method gives better solutions compared with other methods, although the other methods outperforms ours in a few instances, but in most instances our report is the most interesting performance of others.

The graphs (Figures 3, 4, 5 and 6) illustrate with curves representing the difference in quality between the different methods compared for ten taillard's instances (Ta01-Ta28)

For the second runs we enlarged the instance above noted by transportation and empty moving times as follows:

We consider the empty time are equal to the transportation time, and the transportation time between machine $\mathrm{h}$ and machine $\mathrm{k}$ is; $\mathrm{tr}_{\mathrm{hk}}=6^{*}|\mathrm{~h}-\mathrm{k}|$, we take into account the initial $(\mathrm{h}=0)$ and final deposit $(\mathrm{h}=\mathrm{m}+1)$. In this case, to construct one scheduling we use two ant, each one is lied to one transporter robot and each ant represent one of transporter robot.

Displacement of each ant is taking into consideration the additional constraints that support the transport of tasks between machines, each ant moves along the transition probability: 3”)

Where $d_{i j}$ is the distance between machine $i$ and machine $\mathrm{j}$, LC the candidate list and $\tau_{i j}, \eta_{i j}$ and $\theta_{i j}$ is denoted above.

$p_{i j}= \begin{cases}\frac{\left\{\left(\tau_{i j}\right)^{\alpha} \times\left(\eta_{i j}\right)^{\beta} \times\left(\theta_{i j}\right)^{\varphi} \times\left(d_{i j}\right)^{\delta}\right\}}{\sum_{j \in L C}\left(\tau_{i j}\right)^{\alpha} \times\left(\eta_{i j}\right)^{\beta} \times\left(\theta_{i j}\right)^{\varphi} \times\left(d_{i j}\right)^{\delta}} & \text { if } j \in L C \quad \text { otherwise } \\ 0 & \text { otherwise }\end{cases}$

The two ants communicate between them in the sense that an ant cannot take the destination of a machine unless the other ants do not take as a destination, or that the other ants arrive at the desired machine too late that the other ant.
The Table 2 shows the computational result of the Flow shop problem with the transportation constraints and the limited buffers connected at each machine can only support one task at a time, and the robot transporter can only transports only one task at a time.

\section{ACO Pseudo code for flow shop problem with transportation times:}

1. Initialization: The pheromone trails, the heuristic information and the parameters are initialized

2. Iterative loop:

2.1 Each ant of the colony is positioned in the initial deposit.

2.2 Each two ants from the colony construct a complete schedule:

Ant1 construct the path make by the first robot

Ant2 construct the path make by the second robot

/* The construction of the both paths is made parallel by the two ants while communicating with each other */

Repeat

- Construct a list of candidates

- For each ant:

o Apply state transition rule (3',) to select the next processing job in the candidate list

o Apply the local updating rule

Until a complete schedule is constructed

2.3 Apply the global updating rule

3. Cycle: If the maximum number of iterations is realized, then STOP;

Else go to step 2.

Unfortunately, for this kind of problems: flow shop with transportation times, there is no instance neither any computational result. So to validate our method ACO, we were obligated to enlarge the taillard's benchmark by the moving and empty times. We assume that the machines are distanced by identical distance $\mathrm{d}_{\mathrm{hk}}=|\mathrm{h}-\mathrm{k}| * \mathrm{D}$, where $\mathrm{h}$ and $\mathrm{k}$ are the index of machines and $\mathrm{D}$ is the time unit taken by one transporter robot to travel between two consecutive machines, in our runs we suppose $D=6$ for both moving and empty times. And we reduce the buffers capacity of machines to one task at a time, the results shown in Table 2 present the results of runs of each first instance of each type of taillard's benchmark. In the table above we give the solution of classical PFSSP found in the literature, and in the right column we give our solution for our typical problem studied in this paper. 
Table 1. Makespan comparison

\begin{tabular}{|lllllllll}
\hline N_ $\mathrm{M}$ & $\begin{array}{l}\text { Problem } \\
\text { number }\end{array}$ & HAMC1 & HAMC2 & HAMC3 & CR(MC) & GA & MOACSA & ACOC \\
\hline $20 \_5$ & ta001 & 1.49 & 3.60 & 2.27 & 6.34 & 0.00 & 1.49 & $0.16^{*}$ \\
\hline $20 \_5$ & ta002 & 0.00 & 2.62 & 2.62 & 0.36 & 0.00 & 0.73 & 0.81 \\
\hline $20 \_5$ & ta003 & 5.79 & 6.14 & 6.14 & 7.89 & 0.00 & 5.53 & $4.63^{*}$ \\
\hline $20 \_5$ & ta004 & 4.71 & 6.27 & 5.90 & 4.03 & 0.00 & 2.91 & $0.64^{*}$ \\
\hline $20 \_5$ & ta005 & 2.07 & 6.12 & 6.12 & 0.00 & 1.61 & 0.31 & 0.89 \\
\hline $20 \_5$ & ta006 & 0.00 & 3.47 & 3.47 & 3.55 & 2.99 & 0.57 & 2.59 \\
\hline $20 \_5$ & ta007 & 3.28 & 6.17 & 4.06 & 8.36 & 0.00 & 1.88 & $1.69 *$ \\
\hline $20 \_5$ & ta008 & 1.74 & 10.99 & 10.99 & 6.25 & 4.35 & 0.00 & 2.07 \\
\hline $20 \_5$ & ta009 & 0.31 & 6.06 & 6.06 & 2.46 & 2.30 & 0.00 & 2.60 \\
\hline $20 \_5$ & ta010 & 1.36 & 10.09 & 3.56 & 1.02 & 1.95 & 0.00 & 2.17 \\
\hline & & & & & & & & \\
\hline $20 \_10$ & ta011 & 6.16 & 8.44 & 6.94 & 2.39 & 0.00 & 0.66 & 6.38 \\
\hline $20 \_10$ & ta012 & 2.34 & 3.83 & 4.69 & 9.49 & 4.17 & 0.00 & 6.09 \\
\hline $20 \_10$ & ta013 & 5.59 & 14.65 & 14.59 & 3.92 & 3.15 & 0.00 & 5.35 \\
\hline $20 \_10$ & ta014 & 2.68 & 6.98 & 6.24 & 2.82 & 0.20 & 0.00 & 7.04 \\
\hline $20 \_10$ & ta015 & 15.57 & 4.50 & 6.44 & 6.58 & 1.54 & 0.00 & 6.83 \\
\hline $20 \_10$ & ta016 & 4.61 & 8.63 & 8.18 & 1.56 & 0.00 & 1.49 & 6.87 \\
\hline $20 \_10$ & ta017 & 0.95 & 2.85 & 3.10 & 2.72 & 0.00 & 0.70 & 4.85 \\
\hline $20 \_10$ & ta018 & 2.19 & 3.95 & 3.95 & 12.78 & 2.82 & 0.00 & 5.00 \\
\hline $20 \_10$ & ta019 & 5.74 & 10.10 & 8.97 & 2.63 & 0.00 & 1.02 & 3.95 \\
\hline $20 \_10$ & ta020 & 1.45 & 6.52 & 4.30 & 6.52 & 1.75 & 0.00 & 6.54 \\
\hline & & & & & & & & \\
\hline $20 \_20$ & ta021 & 2.51 & 4.49 & 4.77 & 7.41 & 0.45 & 0.00 & 5.44 \\
\hline $20 \_20$ & ta022 & 11.96 & 11.96 & 16.22 & 3.42 & 0.00 & 2.56 & 5.43 \\
\hline $20 \_20$ & ta023 & 0.46 & 0.91 & 3.94 & 0.00 & 7.13 & 4.31 & 6.06 \\
\hline $20 \_20$ & ta024 & 11.41 & 16.88 & 18.14 & 7.25 & 0.00 & 0.30 & 6.70 \\
\hline $20 \_20$ & ta025 & 0.00 & 1.36 & 3.02 & 0.29 & 0.21 & 2.19 & 7.90 \\
\hline $20 \_20$ & ta026 & 9.04 & 12.62 & 13.56 & 5.16 & 0.17 & 0.00 & 6.33 \\
\hline $20 \_20$ & ta027 & 12.60 & 16.28 & 15.69 & 0.00 & 10.40 & 9.38 & 5.67 \\
\hline $20 \_20$ & ta028 & 5.12 & 5.50 & 5.46 & 3.16 & 0.00 & 3.16 & 6.07 \\
\hline & & & & & & & & \\
\hline
\end{tabular}

Table 2. Computational result of the PFSP

\begin{tabular}{|lllll|}
\hline Instances & Number of jobs & $\begin{array}{l}\text { Number } \\
\text { machines }\end{array}$ & $\begin{array}{l}\text { Best solution known } \\
\text { Without transportation }\end{array}$ & $\begin{array}{l}\text { Our solution with Transport and } \\
\text { limited buffers }\end{array}$ \\
\hline ta001 & 20 & 5 & 1278 & 1487 \\
\hline ta011 & 20 & 10 & 1582 & 2134 \\
\hline ta021 & 20 & 20 & 2297 & 3469 \\
\hline ta031 & 50 & 5 & 2724 & 3119 \\
\hline ta041 & 50 & 10 & 3025 & 4036 \\
\hline ta051 & 50 & 20 & 3875 & 7080 \\
\hline ta061 & 100 & 5 & 5493 & 6245 \\
\hline ta071 & 100 & 10 & 5770 & 7563 \\
\hline ta081 & 100 & 20 & 6282 & 13270 \\
\hline ta091 & 200 & 10 & 10862 & 14188 \\
\hline
\end{tabular}




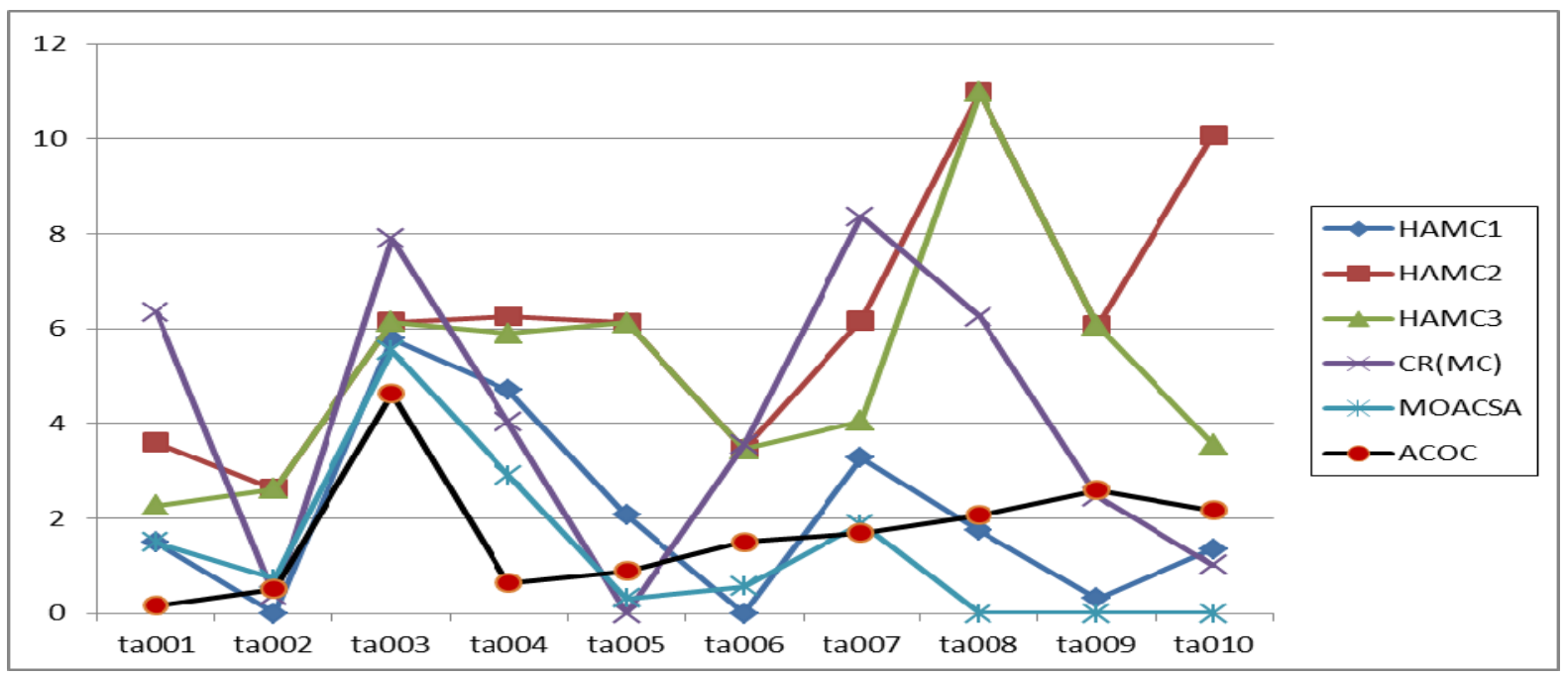

Figure 3. Index of performance comparison for taillard's instance (Ta01-Ta10)

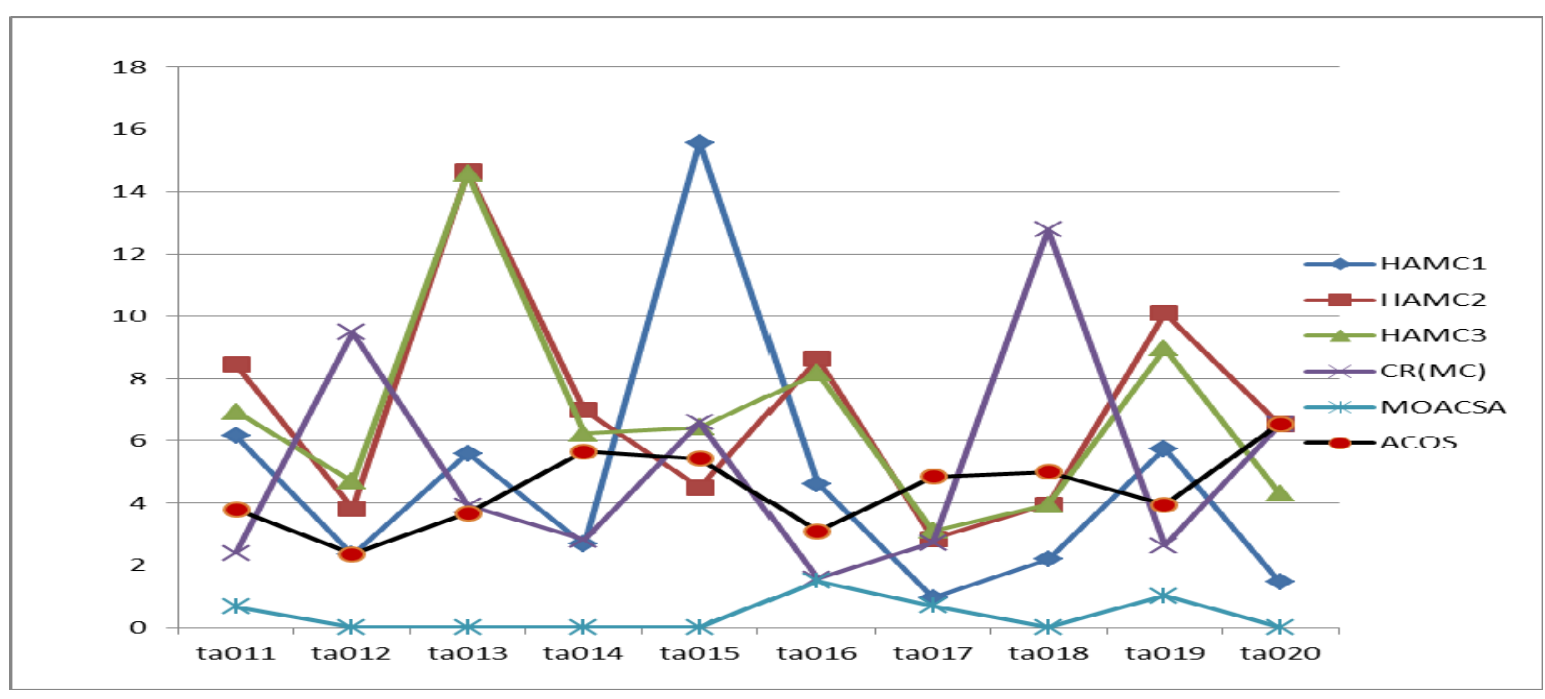

Figure 4. Index of performance comparison for taillard's instance (Ta11-Ta20)

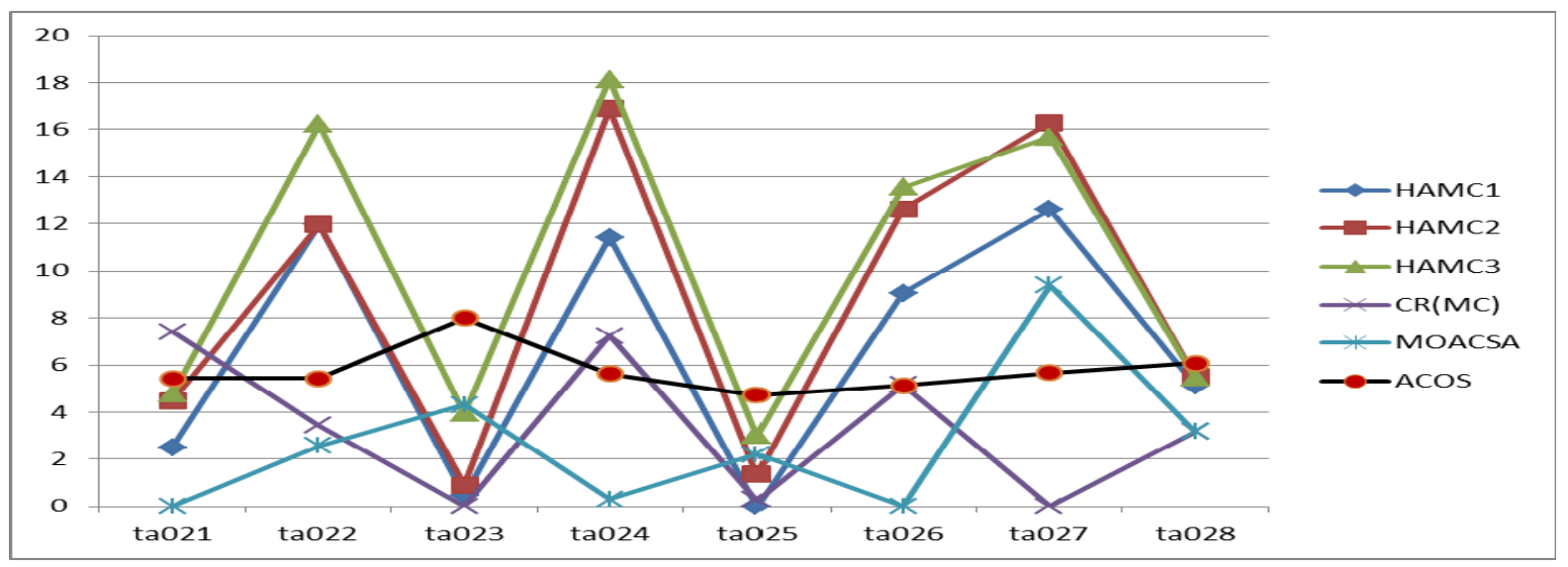

Figure 5. Index of performance comparison for taillard's instances (Ta21-Ta28) 


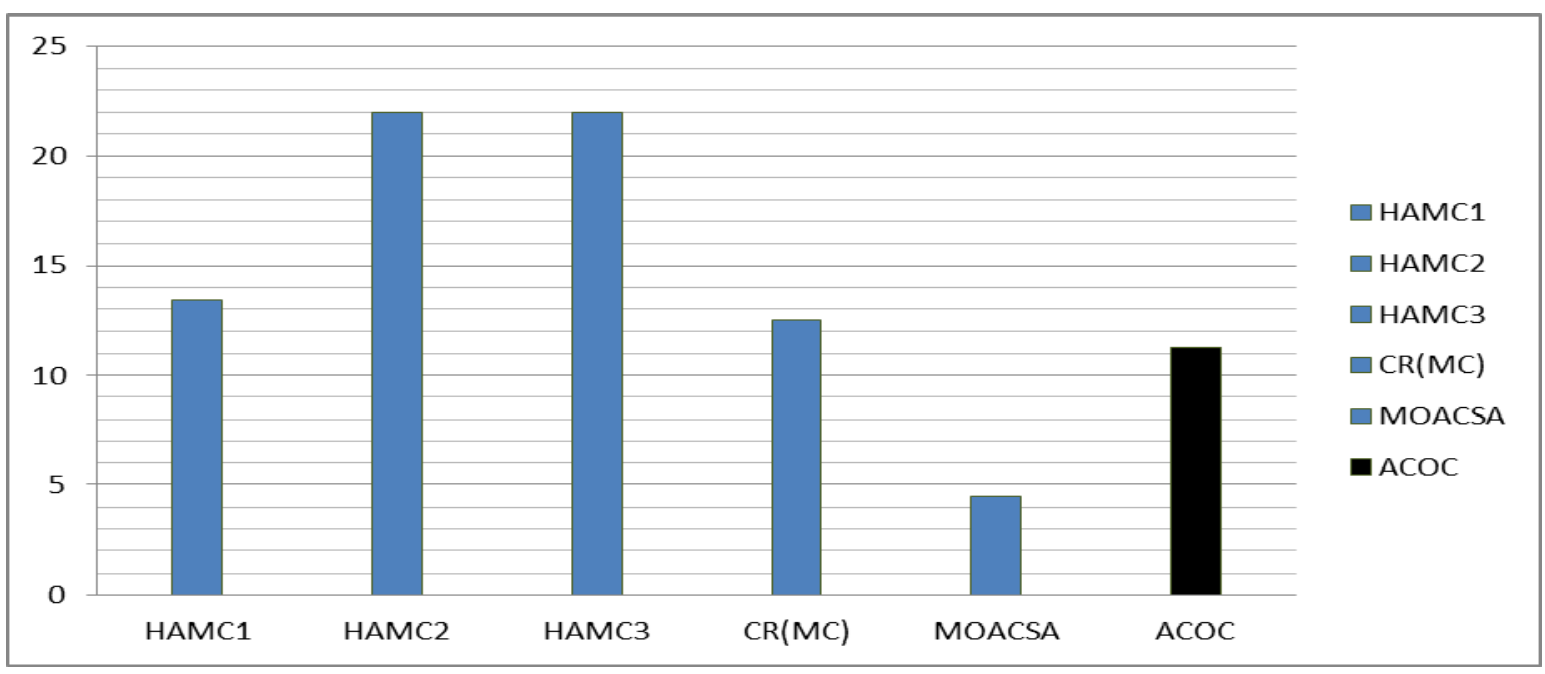

Figure 6. The average index of performance comparison for taillard's instances (Ta01-Ta28)

\section{Conclusion}

The majority of the studies about flow-shop scheduling have been mainly focused on a classical problem without take into account transportation times. However, many real-world problems require solution approaches which take into account moving and empty time arising from the displacement of tasks between machines. For this reason, recent researches are tending to find solution approaches for the problem with the transportation time constraints. Hence, we consider the flow shop scheduling problem with the transportation time in this study. In this paper, the proposed algorithm is based on ACO metaheuristic. In order to verify the performance of proposed algorithm, computational experiments are conducted on the benchmark problems. The obtained results show that proposed ACO performs better than MOACSA algorithm, CR(MC) algorithm, HAMC algorithms and GA for the multi-objective flow shop scheduling problem. MOACSA can be used for single or multiple objectives case considering different criteria like mean flow time, total tardiness, and maximum tardiness of this problem and it is strongly expected that good solutions will be obtained. Furthermore, it is possible that the proposed ACO algorithm can be applied to the scheduling problems in various manufacturing systems such as job shop, cellular manufacturing, and flexible manufacturing.

\section{References}

[1] S. P., Bansal, 'Minimizing the sum of completion times of n-jobs over m-machines in a flowshop - A branch and bound approach”'. AIIE Transactions, 9, 306-311, 1977.
[2] P. Brucker, S. Heitmann, J. Hurink, '’Flow-shop problems with intermediate buffers", OR Spectrum 25, 2003, 549-574.

[3] A. Colorni, M. Dorigo, F. Maffioli, V. Maniezzo, G. Righini, M. Trubian, 'Heuristics from nature for hard combinatorial optimization problems', International Transactions in Operational Research, Volume 3, Issue 1, January 1996, Pages1-21.

[4] Dawande M. N., Geismer H. N., Sethi S. P. \& Sriskandarajah C., ''Throughput optimization in robotic cells”, Berlin: Springer, 2007.

[5] Gambardella, L. M., \& Dorigo, M., 'Solving symmetric and asymmetric TSPs by ant colonies", In Proceedings of the 1996 IEEE international conference on evolutionary computation. Piscataway: IEEE Press, 1996, pp. 622-627.

[6] M. Garey, D.S. Johnson, R. Sethi, 'The complexity of flow-shop and job-shop scheduling”', Math. Oper. Res. 1, 1976, 117-129.

[7] R. Graham, E.L. Lawler, J.K. Lenstra, A.H.G. Rinnooy-Kan, "Optimization and approximation in deterministic sequencing and scheduling: a survey', Ann. Discrete Math. 5, 1979, 287-326.

[8] J., Hurink, S., Knust, 'Makespan minimization for flow-shop problems with transportation times and a single robot.", Discrete Applied Mathematics, Volume 112, Issues 1-3, 15 September 2001, Pages 199-216.

[9] H. Kise, 'On an automated two-machine flowshop scheduling problem with infinite buffer', J. Oper. Res. Soc. Japan 34, 1991, 354-361.

[10] H. Kise, T. Shioyama, T. Ibaraki, 'Automated twomachine flowshop scheduling: a solvable case', IIE Trans. 23, 1991, 10-16. 
[11] E. Nowicki, ''The permutation flow shop with buffers: a tabu search approach', European Journal of Operational Research 116, 1999, 205-219.

[12] B., Qian, L., Wang, De-xian Huang, Wan-liang Wang, Xiong Wang, "'An effective hybrid DE-based algorithm for multi-objective flow shop scheduling with limited buffers”, Computers \& Operations Research, Volume 36, Issue 1, January 2009, Pages 209-233.

[13] S.S. Panwalkar, "Scheduling of a two-machine flowshop with travel time between machines', J. Opl. Res. Soc. 42, 1991, 609-613.

[14] C., Rajendran, ' 'Heuristics for scheduling in flowshop with multiple objectives'” European Journal of Operational Research, 82, 1995, 540-555.

[15] Ravindran D., Noorul Haq A., Selvakuar S. J. \& Sivaraman R., "Flow shop scheduling with multiple objective of minimizing makespan and total flow time", International Journal of Advanced Manufacturing Technology, 25, 2005, 1007-1012.

[16] H. Stern, G. Vitner, '’Scheduling parts in a combined production-transportation work cell', J. Opl. Res. Soc. 41, 1990, 625-632.

[17] R.F. Tavares Neto, M. Godinho Filho, 'An ant colony optimization approach to a permutational flowshop scheduling problem with outsourcing allowed", Computers \& Operations Research, Volume 38, Issue 9, September 2011, Pages 1286-1293.

[18] E. Taillard, 'Benchmarks for basic scheduling problems', European Journal of Operational Research 64, 1993, 278-285.

[19] B., Yagmahan, M. M., Yenisey., '’A multi-objective ant colony system algorithm for flow shop scheduling problem', Expert Systems with Applications, Volume 37, Issue 2, March 2010, Pages 1361-1368.

[20] KC. Yinga., CJ. Liao., 'An ant colony system for permutation flow-shop sequencing", Computers and Operations Research 31, 2004, 791-801. 